\title{
Pathology measurements closer to the patient?*
}

\author{
K WIENER
}

From the Department of Clinical Biochemistry, North Manchester General Hospital, Crumpsall, Manchester M8 6RB, UK

\section{Historical background}

When pathological testing was in its infancy, the performance of the tests, which were usually fairly simple and limited in range, fell mainly into the domain of the junior doctor and the nursing staff. The procedures were often qualitative or only semiquantitative, requiring a minimum of apparatus, and could be carried out in the side-room of the ward, that is, relatively close to the patient. However, as scientific advances were made and applied to the study of disease, a wider range of tests was needed. Newer and better techniques were introduced to replace the old ones and to supplement them, and the side-room could no longer cope alone with the investigation of the patients on the ward. More specialised scientific knowledge and expertise began to develop within pathology departments, which gradually took over from the side-room as the main provider of pathology measurements, taking tests a little further away from the patient but still usually within the hospital.

The procedures employed in pathology laboratories were traditional manual techniques until the 1960 s, when the first generation of automatic analysers was introduced. Since then the development of automatic instrumentation for pathology measurements has been rapid and continuous to the point where pathology laboratories have, with some justification, been likened to factories employing production-line techniques. The improvements in design of equipment have facilitated faster handling of samples but have inevitably been associated with increases in capital costs of equipment. This increased analytical capacity meant that one laboratory could process samples from other hospitals as well as its own, and economic considerations led to rationalisation and centralisation of pathology services in most areas of the United Kingdom. These changes resulted in the closure of some smaller laboratories with concentration of

*This essay was the winner in the 'under 35 section' of the 1979 Technicon Award.

Received for publication 15 January 1980 resources at the larger centres, a move that took pathology measurements yet another step away from many patients. It also introduced other problems, notably the organisation of transport facilities for samples and reports between hospitals and the district laboratory, which might be situated several miles from some of the beds it served.

The current situation is substantially the one just described, with pathology services relatively close to some patients, ie, those within the same hospital, and quite remote from others. An arrangement such as this is likely to offer a poorer service to the patients situated furthest from the laboratory unless a frequent and efficient transport system is in existence, and this can be a considerable financial burden, particularly if taxis are used to any significant extent for the transport of urgent specimens.

Another disadvantage of the large, centralised laboratory is that the service is likely to be dependent on a small number of large-capacity automatic analysers, and, if one of these breaks down, a large backlog of work builds up quite rapidly. There is therefore something to be said for having a more widely spread analytical capacity so that less reliance need be placed on any one instrument.

\section{Suitable sites outside the laboratory}

\section{SPECIALISED UNITS}

For many years there has been a tendency for specialised clinical units to take an interest in making their own pathology measurements for parameters of particular relevance to their patients. This has applied especially in teaching hospitals where research was being performed and where money was available for the appointment of appropriate scientific or technical staff for this purpose. However, with the formation of intensive care units in district general hospitals, together with the development of clinical measurement techniques, some units there have also found it desirable to perform certain pathology measurements themselves to assist in monitoring their patients. There are obvious advantages in doing this. Specimens can be taken when it is convenient for the staff of the unit and appropriate 
to the needs of the patient, rather than being limited by the time schedules of the laboratory. There are no transport problems and the results are generally available more quickly than those from the laboratory, allowing prompt adjustments to be made to the patient's treatment, if necessary. From a financial point of view, there may also be some advantages in that payments to laboratory staff for duties outside normal hours are avoided, although this saving would be offset if, as a consequence, additional staff were required in the intensive care unit, and also by capital and running costs of equipment required for the unit.

The measurements of particular relevance in an intensive care unit are likely to be electrolytes, urea, blood gases, acid-base parameters, and glucose. When considering what type of staff are appropriate to perform such measurements in intensive care units, it is difficult to generalise because it depends on the type of equipment to be used and on the ability and adaptability of individual staff who are unlikely to have a thorough background training in this sort of work. Of course, laboratory-trained people could be employed specifically for this purpose but it would make a significant addition to the costs.

Some intensive care units initially set out to train nursing staff to perform such analyses but subsequently found it to be unsatisfactory, partly because of a lack of technical expertise on the part of the trainees and partly because of the speed of turnover of nursing staff. Some units have therefore reverted to relying on the hospital laboratory for all their pathology measurements.

Another specialised unit that may find it convenient to perform some of its own pathology tests is the special care baby unit. Plasma bilirubin and blood glucose would be among the most appropriate and feasible for such a unit. Bilirubin could be measured spectrophotometrically using a bilirubinometer; stick tests in combination with an appropriate meter could be used to give a quantitative estimate of glucose concentration. Both procedures are designed for use with small capillary samples, and their use eliminates the delays associated with collection of blood by laboratory staff. This type of blood glucose monitoring system can be used in other situations and will be discussed again later.

\section{OUTPATIENT CLINICS}

One area in which taking pathology measurements closer to the patient can result in better patient care is in outpatient clinics, although the scope is rather limited. An obvious example is the monitoring of glucose levels in diabetic outpatients. It is advantageous to have glucose measurements available before the clinician sees the patient, so that any necessary alterations in therapy can be made immediately. This, of course, has long been the practice in many hospitals; in some cases analyses are actually performed in the outpatient department. A similar situation exists for the monitoring of patients on anticoagulant therapy. With the advent of rapid and simple enzyme-linked techniques for the assay of certain anticonvulsant drugs, this principle can now be applied to patients attending epileptic clinics, so that adjustments can be made to dosage on the spot, avoiding the effort and cost of informing patients by letter of any changes needed.

\section{GENERAL WARDS}

The scope for taking pathology measurements closer to the patient on the general hospital ward is still rather limited. It is common practice to perform a wide range of tests on almost every inpatient, and these tests are most economically done within the pathology department. However, it can be argued that many of these tests are unnecessary and that a more selective approach would be more sensible; ${ }^{1}$ but even then it is difficult to envisage routine pathology testing leaving the laboratory to be performed on the wards. There are systems under development, for example, the Kodak Ektachem system, that will enable analyses to be made without the use of wet chemical reagents and that should be suitable for performance by relatively unskilled staff. However, it requires a special spectrophotometer for quantitation, and the cost of materials per test result will probably be quite high.

\section{CASUALTY DEPARTMENTS}

It can be important in casualty departments to have ready access to certain pathological measurements. Some may be available at all times from the laboratory; others may not or may take time to organise, and it is this type of measurement that it would be desirable to have available within the casualty department. However, most procedures are too complicated to be performed under these conditions by untrained staff. Nevertheless, attempts have been made to introduce some analytical procedures; for example, a kit has been marketed for the measurement of paracetamol in blood, the intention being that this could be used at the bedside without the need for any other equipment. A blood alcohol estimation can also be helpful in the investigation of semiconscious and unconscious patients, and instruments are now available which can do this by breath analysis. This enables a rapid estimation to be carried out within the casualty department.

PATHOLOGY MEASUREMENTS BY PATIENTS The ultimate in taking pathology measurements 
closer to the patient would be to allow the patient to perform the tests himself. In fact, this is being done in some places for certain tests. In order to assist in the monitoring and control of their condition, diabetics have long performed qualitative and semiquantitative tests on samples of their urine for reducing substances and ketones. Moreover, recent studies have indicated that selected diabetic patients can achieve better control of their condition if they are allowed to monitor their own blood glucose levels. ${ }^{2} 3$ This is now possible owing to the introduction of relatively inexpensive meters for quantitating the colour responses of stick tests for blood glucose (Fig. 1). The patient is required to prick his finger, to apply blood correctly to the test strip for a timed interval, and to use the instrument according to a set of fairly simple instructions. Of course, not all patients are capable of doing this but those who are, and who have sufficient motivation to do it conscientiously, can achieve stabilisation at home without the need to occupy hospital beds. Although the accuracy and precision of such procedures are inferior to those of most laboratory methods, they can be adequate for this purpose.

Participation in this way gives the patient a better understanding of his condition and, one hopes, a greater incentive to control it. Obviously, it is important to select patients carefully for selfmonitoring and to avoid both those who would find it technically too demanding and those who would develop a morbid interest in self-assessment.

There are also some occult blood testing kits on the market which allow for a degree of patient participation. The patient is required to apply a small portion of faeces to a reagent-impregnated paper which is then resealed in a wallet-type container before being forwarded to the clinician or other interested person, who reopens it and completes the test by adding developing reagent and observing the colour change.

\section{Technical, financial and managerial aspects}

\section{ANALYTICAL CONSIDERATIONS}

The movement of pathology measurements closer to the patient often means that tests will be performed by personnel less skilled in analytical techniques, and therefore the procedures employed must be as simple and straightforward as possible. Attempts have been made by equipment manufacturers to achieve this by making their instruments operate as automatically as possible. A good example of this is blood gas analysers, some of which now calibrate themselves automatically at regular intervals. This approach has certain advantages, but it can also be rather restrictive to the analyst; for example, in the

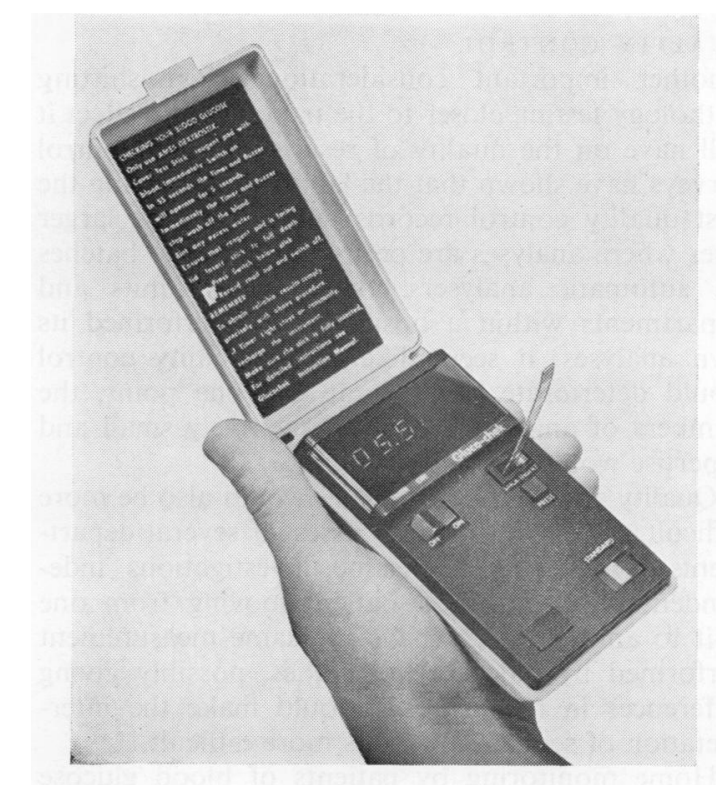

(a)

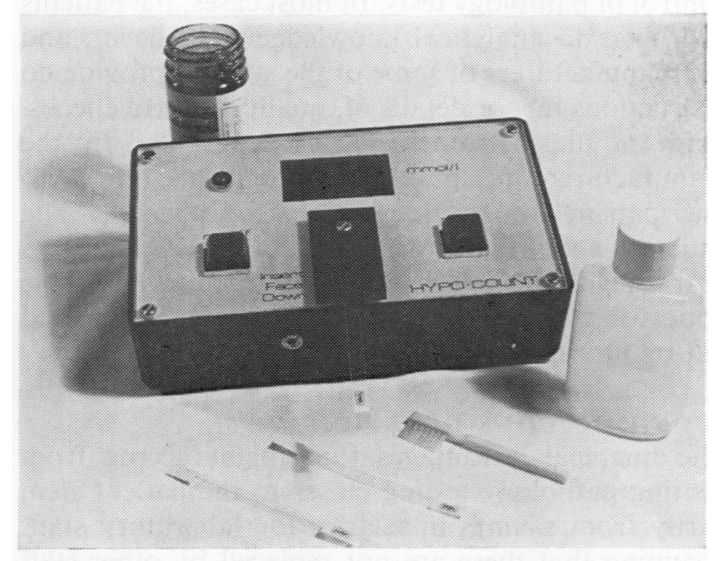

(b)

Fig. 1 Two examples of meters specifically designed for use by patients at home for monitoring blood glucose: (a) Gluco-chek by Medistron Ltd, and (b) Hypo-count by Hypoguard Ltd.

case of patented reagent systems, the procedure is dictated by the manufacturer, and the analyst has no control over it whatsoever. It is a fact that the simplest chemical procedures are frequently not the most specific or precise for the measurement of constituents in biological fluids, and there is a danger that analytical desirability may be sacrificed for convenience. 
QUALITY CONTROL

Another important consideration when shifting pathology testing closer to the patient is the effect it will have on the quality of results. Quality control surveys have shown that the laboratories having the best quality control records tend to be the larger ones where analyses are performed in large batches on automatic analysers. If individual units and departments within a hospital each performed its own analyses, it seems likely that quality control would deteriorate because, at any one point, the numbers of analyses would be relatively small and expertise would be relatively limited.

Quality control performances would also be more difficult and more costly to assess if several departments were doing the same investigations independently. Moreover, a patient moving from one unit to another might have the same measurement performed by different techniques, possibly giving differences in results. This would make the interpretation of sequential results more difficult.

Home monitoring by patients of blood glucose levels opens up a whole new aspect of the quality control of pathology tests. In most cases, the patients will have no analytical knowledge whatsoever, and the manufacturers of some of the systems provide no instructions for, or details of, quality control checks. Some of the instruments are precalibrated by the manufacturer, and there may be no means offered to the patient for checking this periodically. The situation should improve with further developments in this field but there is still likely to be considerable opportunity for error when analyses are being carried out by untrained amateurs.

\section{ECONOMIC CONSIDERATIONS}

The financial advantages that might accrue from resiting pathology testing closer to the patient stem partly from savings in salaries for laboratory staff, assuming that these are not replaced by other staff elsewhere. There would also be savings in capital costs of large-capacity automatic analysers although this would be offset by the costs of larger numbers of less expensive instruments to be sited nearer to the patients. A further saving would result from the elimination of sophisticated and expensive data processing systems that are currently in use in hospital laboratories for the handling of test information and reporting of results. Economies would also ensue from avoidance of expensive transport systems for samples and reports.

On the debit side, reagent costs would probably be higher because they would be used less economically and might often be in a specially prepared commercial form, for example, test strips, tablets, and dry films. Assuming quality control procedures were extensively applied, costs for control materials would be higher owing to the performance of tests in small batches, each with its control samples.

It is probably reasonable to predict a reduction in the total number of tests performed because clinicians would be more selective in their requests if individual tests were to be processed on or near the ward rather than in the laboratory as, for example, biochemical 'profiles'. Economies may therefore result in this way. In the case of home glucose monitoring by patients, the savings in occupancy of hospital beds could more than pay for the monitoring equipment, provided that the latter was used wisely and fully. There would also be savings in hospital staff time with respect to collection and analysis of blood samples.

\section{HEALTH AND SAFETY}

With the coming of the Health and Safety at Work Act (1974) and the Howie Report, ${ }^{4}$ considerable attention has been paid to the tightening up of safety procedures within pathology departments and to the formulation of rules governing the handling of certain types of specimen. If pathology tests were to be performed at numerous sites throughout the hospital, the hazards associated with high-risk specimens of biological material would be even greater than at present. The handling and analysis of such materials is best carried out by experienced staff in specially equipped rooms away from patient areas. In general, the safety aspects of pathology testing might pose problems in a system dissipated throughout the hospital.

MANAGEMENT AND STAFFING ASPECTS

Financial matters have already been dealt with and will not be elaborated here, but other aspects of management must be borne in mind. If pathology testing were being performed outside the laboratory to any significant extent, then the pathology laboratory as we now know it would change markedly. In fact, some sections of it may not be needed at all.

It has already been suggested that the number of laboratory staff might be reduced. This would have serious implications for laboratory staff in general in the short term, but especially so in the long term for those who see laboratory medicine as a lifelong career and for those already in senior posts who would see their department's, and consequently their own, responsibilities diminish, unless they were retained in a consultative and training capacity to provide expert advice on pathology testing procedures outside the laboratory and to supervise the training of the staff who would use them.

For the most part in this essay it has been assumed 
that pathology testing outside the laboratory would be performed by the staff of the unit or ward but, conceivably, staff could be sent out from the laboratory to carry out the tests on the wards. This would seem, however, to be a less efficient way of doing things. Whatever the final arrangements, someone would have to shoulder the responsibility of choosing the procedures to be employed, deciding on the type of equipment to be purchased, ensuring that the analytical procedures were performed properly, and monitoring the quality of the results. These could all be done unilaterally by staff within the individual units, but it seems more sensible to allow an expert to take an overall view of the situation, and such a person is most likely to be found in the pathology department.

Whatever happens, it is probable that certain tests will remain within the pathology department, so that the nucleus of expert knowledge relating to pathology tests would also remain there. Nevertheless, even if it were accepted that extralaboratory pathology testing should be under the control of the laboratory, it would still be difficult to implement effectively owing to geographical considerations and, perhaps more important, to the probability that the staff involved would themselves not be under the direct control of the laboratory.

\section{General prospects}

The trend in pathology instrumentation in recent years has been towards more sophisticated instruments that are designed for simplicity of use. A significant contribution to this has been made by advances in electrode technology, so that more selective measurement of certain constituents of biological fluids can be made without resorting to complicated multistage chemical separations or reactions, and without the need for special services other than a supply of electricity and possibly a drain.

Improvements in electronic circuitry, together with a move to producing instruments specifically for a particular measurement, have resulted in direct-reading digital display models, which avoid the need for the operator to perform calculations. Indeed, in procedures where derived values are required, such as in blood gas analysis, there is now usually built into the instrument a calculator, which performs the appropriate computations.

The microprocessor revolution is already beginning to affect pathology laboratories in that some new instruments are being marketed with microprocessor control. This should make it even easier for a relatively unskilled operator to handle sophisticated pieces of equipment. Step-by-step instructions can be given to the operator, and, if these are not followed correctly, error messages can be delivered. In conjunction with the largely automatic preprogrammed operation that is possible with microprocessor-controlled units, this should be very effective in eliminating operator error and should contribute to the quality of the results.

Other significant developments have been and are being made in the manufacture of chemical reagent systems, which avoid the use of the traditional wet chemical techniques. Dipstick methods have long been acknowledged in clinical chemistry as generally acceptable, if not always ideal, methods for the qualitative investigation of urine samples. Recent work in this field suggests that before long we might see wider application of this type of technique to quantitative work with blood or plasma. If suitable chemical reactions can be found and satisfactorily incorporated into test strips, it would be a relatively simple matter to produce an appropriate spectrophotometer for quantitation purposes.

The thin-film, multilayer methods currently under development represent an important variant of the dry chemical methods. ${ }^{56}$ Here, undiluted serum is applied to the special slide and allowed to diffuse into it. The number of layers needed on the slide will depend on the constituent to be measured but will include an initial spreading layer to control diffusion of analyte and perhaps to filter high molecular weight compounds before passing to a reagent layer where an appropriate chemical reaction is effected. Other selective membranes can be included to control movement of reaction products to further reagent layers. Finally, quantitation by reflectance spectrophotometry can be performed in a suitable instrument (Fig. 2).

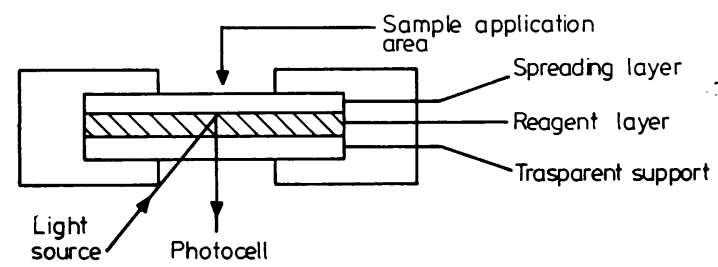

Fig. 2 Diagrammatic representation of a cross-section through the Kodak Ektachem reagent slide in its simplest form.

It is progress in the above-mentioned fields that is likely to facilitate the movement of pathology testing closer to the patient, and it is worthy of note that this progress is almost entirely dependent on developments in commercial organisations. This is particularly true of the dry chemical techniques where, once committed to such a system, the user is 
obliged to employ the chemical procedure determined by the manufacturers for a particular test and to buy the test materials from them at their prices, as the only supplier.

In spite of these developments to make pathology testing sufficiently simple for the user that it can be taken out of the laboratory environment and resited nearer the patient, it will be a long time before the laboratory becomes redundant. As yet, it is a relatively small number of analyses that have been successfully applied in this way. The tests that have been studied most intensively are those that are used most frequently and those that are required quickly for the immediate therapy of patients, eg, glucose, electrolytes, urea, and blood gases. These are also the ones that can be measured most quickly by conventional techniques in the laboratory. In contrast, the measurement of hormone levels might pose greater problems. Nevertheless, enzyme-linked techniques are now becoming available which enable certain hormones to be measured fairly quickly without the need for very complicated equipment. In a few years' time it may be the norm to have methods for thyroid hormone testing sited in clinics devoted to the treatment of patients suffering from thyroid disorders. This would enable patients to have their hormone levels measured before being seen by the clinician, thus allowing treatment to be adjusted accordingly, analogous to the arrangement in diabetic clinics.

With the development of suitable sensing devices, the principle of continuous in vivo monitoring may well be applied to patients who need frequent measurements of certain parameters. This would be particularly suited to acutely ill subjects who require constant supervision. For example, in cases of diabetic ketoacidosis it would be very useful to be able to take frequent measurements of $\mathrm{pH}$, sodium, potassium, bicarbonate, blood gases, and glucose at the touch of a button without having to withdraw a quantity of blood each time. Intensive care units are the obvious sites for this, and in the not-toodistant future we may see this sort of information being displayed along with ECG traces on the cathode ray tubes of such units.

Looking outside the confines of the United Kingdom National Health Service, it seems probable that private clinics might find the convenience of the dry chemical techniques very appealing for testing the moderate number of samples that they are likely to handle. It is also conceivable that, within the Health Service, this type of system might find application in some of the community health centres that have been and are being set up. This would avoid transporting samples between these centres and hospital laboratories, giving a quicker service to general practitioners and their patients.

\section{Summary and conclusions}

Continuing improvements in analytical systems are making pathology testing simpler to perform, and modern methods of investigation and treatment tend to require greater support in the form of information from pathology tests. These trends together have encouraged a move towards taking pathology testing closer to the patient. This should enable a quicker service to be given, but it is, of course, directly opposed to the moves towards centralisation which took place a few years ago and represents a return towards the side-room approach to pathology testing. An increased speed of service has obvious implications in terms of patient care, especially for those acutely ill and requiring constant supervision. However, apart from these superficial advantages, the way is far from clear for a wholesale shift of resources to this type of approach. It is necessary to consider the financial obligations of setting up a system for pathology testing and the number of patients who will benefit from it. It is difficult to generalise on this because both capital and running costs must be borne in mind, and the latter can be high for some systems. The saving in laboratory and transport costs must also be taken into account.

A cause for concern with some of the systems is that they depend on reagent materials produced by one manufacturer, making them rigid and possibly expensive. It also means that the choice of method will be made by the manufacturer without reference to the user. Another cause for concern is quality control, which may deteriorate if testing is to be dissipated throughout the hospital.

It is clear that only certain units in a hospital could justify having their own pathology tests available and even then only for certain parameters. This means that the laboratory would probably still have to provide a service for other units, so that duplication of facilities would occur.

In conclusion, the trend towards pathology testing closer to the patient should be viewed with cautious optimism. Each situation should be treated with individual attention. The advantages and disadvantages should be carefully weighed before a decision is taken regarding whether it is better for the laboratory to provide the necessary service or to site the testing elsewhere. The areas that look most attractive for the latter are close monitoring of acutely ill patients, those undergoing detailed investigation over a period of time, some outpatient clinics, casualty departments, and, in certain cases, patients at home. 


\section{References}

${ }^{1}$ Sandler G. Costs of unnecessary tests. Br Med J 1979;2: 21-4.

2 Sönksen PH, Judd SL, Lowy C. Home-monitoring of blood glucose. Lancet 1978;1:729-32.

${ }^{3}$ Walford S, Gale EAM, Allison SP, Tattersall RB. Selfmonitoring of blood glucose. Lancet 1978;1:732-5.

4 Code of Practice for the Prevention of Infection in Clinical Laboratories and Post-mortem Rooms. HMSO;1978.

s Curme HG, Columbus RL, Dappen GM et al. Multilayer film elements for clinical analysis: General concepts. Clin Chem 1978;24:1335-42.

- Spayd RW, Bruschi B, Burdick BA et al. Multilayer film elements for clinical analysis: Application to representative chemical determinations. Clin Chem 1978;24:1343-50.

Requests for reprints to: Dr K Wiener, Department of Pathology, North Manchester General Hospital, Crumpsall, Manchester M8 6RB, UK. 